\title{
Evaluation of round window accessibility for electrode insertion: validation study from two centers
}

\begin{abstract}
Purpose: Round window insertion is advocated as a method to reduce insertion trauma and preserve residual hearing. Bony overhang of the round window niche and variations in the cochlear rotation might hamper the accessibility of round window membrane. This paper is an attempt to classify the round window membrane visibility before any drilling of the round window niche through the standard posterior tympanotomy approach.
\end{abstract}

Methods: A prospective study was carried out in two centers located in different parts of the country between October 2014 and June 2015. 55 patients in one center and 129 patients in another center underwent Cochlear Implantation through a round window approach. The round window membrane visible to the Surgeon through the posterior tympanotomy was graded into 4 types from I to IV based on visibility from $100 \%$ to nil. In all the cases cochlear implantation was accomplished successfully. Cases with abnormal cochlea were excluded.

Results: There was a significant difference between the distributions of grades I, II, and IV in the two centers. However the distribution of Grade III was comparable between the two centers.

Conclusions: The Grade III type round window is the commonest type seen in patients undergoing cochlear Implantation in two geographic regions in India. This classification is surgically viable and aids the surgeon to access the round window membrane.

Keywords: round window membrane, round window accessibility, classification of round window membrane, round window after posterior tympanotomy
Volume 8 Issue 5 - 2017

\author{
Naresh Panda,' Mohan Kameswaran, ${ }^{2}$ \\ Sourabha K Patro,' Saran S, ${ }^{2}$ Gyanranjan \\ Nayak' \\ 'Department of Otolaryngology and Head and Neck Surgery, \\ India \\ ${ }^{2}$ Madras ENT Research Foundation, India
}

Correspondence: Naresh K Panda Professor and Head Department of Otolaryngology and Head and Neck surgery PGIMER Chandigarh, India, Tel +9l-7087009760, Emailnpanda59@yahoo.co.in

Received: May 25, 2017 | Published: September 25, 2017
Abbreviations: RWN, round window niche; RWI, round window intentioned; RWM, round window membrane

\section{Introduction}

The preservation of residual haring has emerged as an issue of concern in cochlear implantation. The indications of cochlear implantation have broadened from bilateral profound sensory neural hearing loss with normal auditory nerve function to include cases with residual hearing. ${ }^{1}$ More emphasis is being given to the study of factors and techniques of preservation of residual hearing in order to maximize the hearing and speech results after cochlear implantation.

The factors that determine preservation of residual hearing are type and dimension of array, ${ }^{2,3}$ traditional cochleostomy vs. round window insertion), ${ }^{4,5}$ insertion technique ${ }^{3,6}$ and drugs used during insertion. ${ }^{7,8}$ Soft surgery technique with round window insertion has played a major part among the steps taken for residual hearing preservation in the last decade. Round window approach to inner ear, reduces insertion trauma and could potentially preserve residual hearing. Hence more surgeons are using the round window approach for Cochlear implantation. While approaching the round window in round window intentioned (RWI) cases, the view of round window membrane is obscured by round window niche (RWN). The visibility of round window membrane after an adequate posterior tympanotomy determines the amount of drilling that is required for accessing the round window for electrode insertion. We propose a classification system for round window visibility after posterior tympanotomy which will guide the surgeon regarding the amount of drilling that may be required for insertion of the electrode. Our classification is different from the earlier proposed St. Thomas hospital classification by Leong et al., ${ }^{9}$ as it classifies round window visibility after adequate drilling of the round window niche.

\section{Methods and materials}

A prospective study was carried out in two tertiary care centers in Northern and Southern part of the country from October 2014 to June 2015. A prior approval of Institute Ethics Committee was obtained. All patients undergoing cochlear implantation during this period in both the institutes were included in the study. There were 184 patients in total including 55 patients in institute 1 and 129 patients in Institute 2. Intra operative round window accessibility was studied in all these patients. Patients with cochlear anomalies detected in preoperative radiological analysis were excluded from the study. The results were analyzed by SPSS v 20.0. (IBM SPSS 2011).$^{10}$ An informed consent was obtained from all the patients (parents in case of minor children) before recruitment.

All the patients were taken up for surgery after a detailed audiological and radiological work up. Cochlear implantation was done with an adequate posterior tympanotomy approach. After achieving the best possible exposure, the round window membrane visibility was graded in order to determine the amount of drilling of RW niche that will be required to perform a round window insertion. This grading was done before any drilling of the round window niche.

The round window visibility was graded as Grade I (Figure 1): $>50 \%$ of the round window membrane is visible. Grade II (Figure 2): $25 \%$ to $50 \%$ of the round window membrane is visible. Grade III 
(Figure 3): $<25 \%$, i.e. only a glimpse of the round window membrane can be seen and Grade IV (Figure 4): Round window membrane is not visible. The same grading system was followed in both the centers uniformly by the two surgeons (author 1 and 2). This classification was used and types and numbers were recorded in both the institutes and later they were compared and correlated for validation. The Personnel who analyzed the data was blinded about the centers.

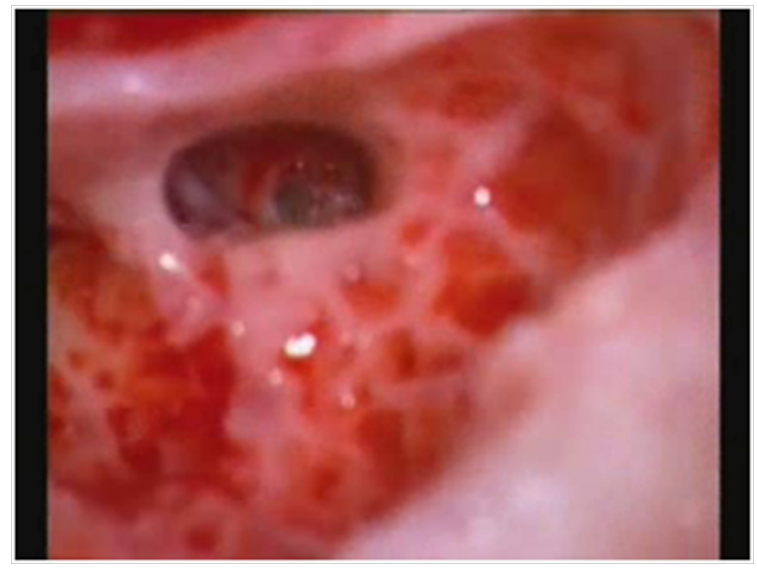

Figure I Grade I round window visibility with > 50\% of the RM membrane being visible.

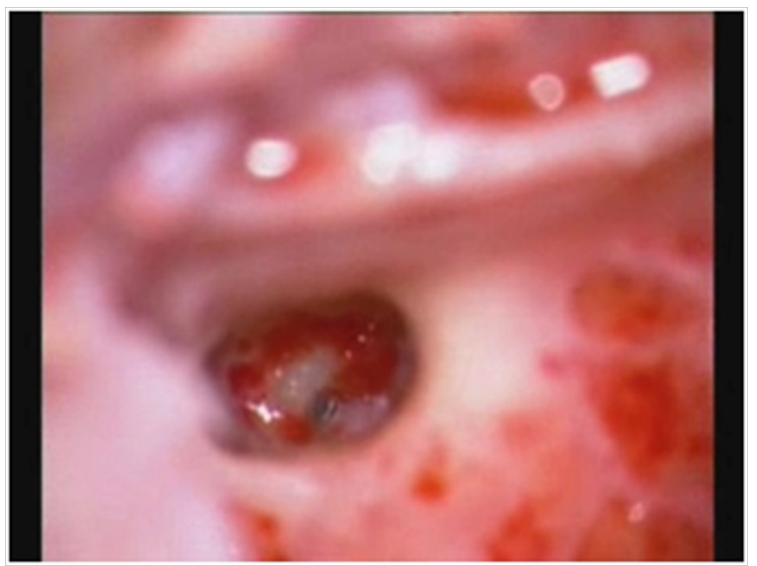

Figure 2 Grade II round window visibility with $25 \%$ - $50 \%$ of the RM membrane being visible.

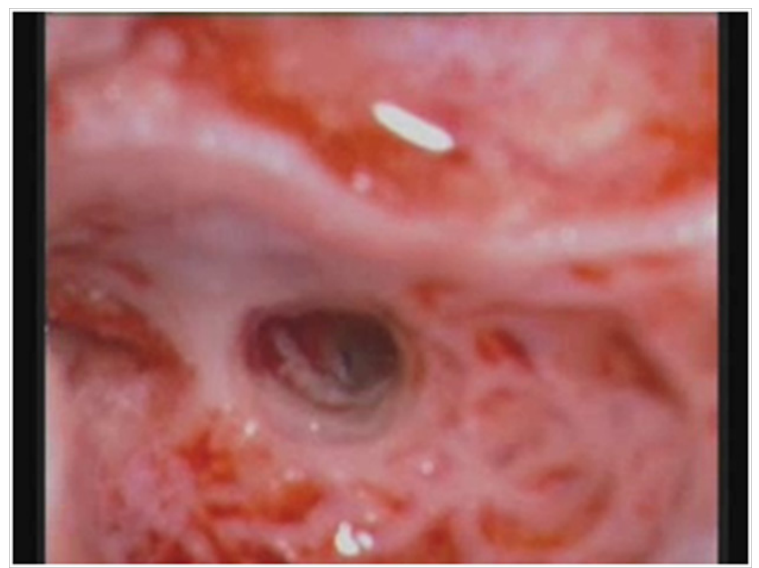

Figure 3 Grade III round window visibility with $<25 \%$ i.e. only a glimpse of the RM membrane being visible.

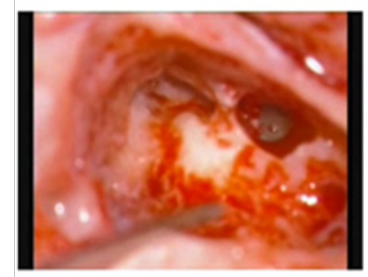

a

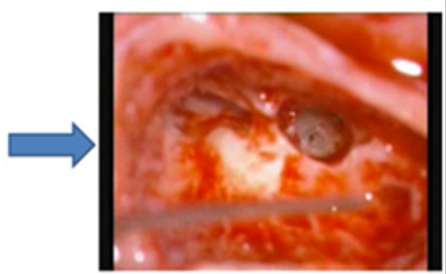

b
Figure 4 a. Grade IV round window visibility with no RW membrane being visible. b: Conventional bony cochleostomy performed with drilling for insertion of the electrode array.

\section{DISTRIBUTION OF GRADES OF ROUND WINDOW}

Types of round window (Institute wise)

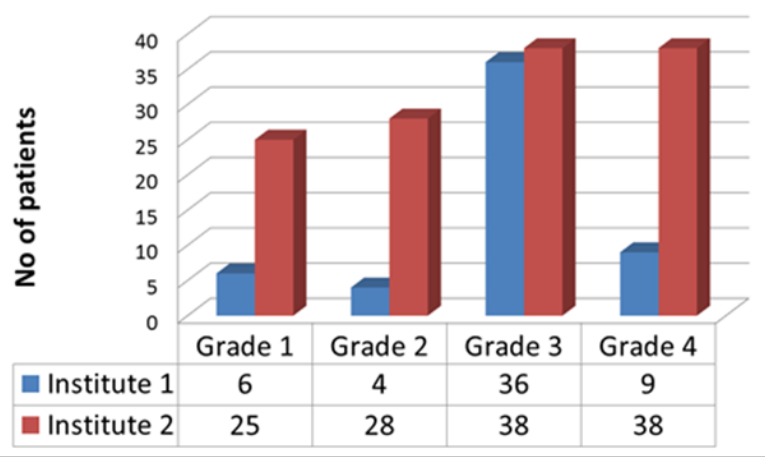

Figure 5 Showing the distribution of RW grades in the two centres.

\section{Results}

There were a total of 55 patients in institute 1 . Out of which grade I, II, III, IV types of round window was seen in $6(10.9 \%)$, $4(7.27 \%), 36(65.45 \%)$ and $9(16.36 \%)$ patients respectively. Institute 2 had 129 patients who underwent cochlear implantation during this period. Of these 129 cases 25(19.37\%), 28(21.70\%), 38(29.45\%) and $38(29.45 \%)$ patients had grade I, II, III and grade IV type of round window membrane visibility respectively. Grade III was the most common type of grade among patient population in both the institutes.(Figure 5) The total distributions of various grades in patient population of both the institutes in two parts of the country were not statistically different from each other $(\mathrm{p}=0.4$, Mann Whitney $\mathrm{U}=3282$, $\mathrm{z}=-0.842$ )

On using $\chi^{2}$ test to compare the individual groups it was seen that both the groups differed significantly in terms of the patient distribution in grade I, II, IV. However, the difference was not significant in grade III (Table 1). Grade III was comparable and was the most common type of round window present in the population in both northern and southern part of the country.

For all the cases with grade I electrode could be easily inserted through the round window. For grade II, minimal drilling of the niche was required for smooth round window electrode insertion. For grade III type of round window complete drilling of round window and cristae fenestra was required. For grade IV type of round window membrane either a round window insertion or a standard cochleostomy anteroinferior to the round window niche was performed. In Institute 13 out 
of $9(33 \%)$ required a standard cochleostomy whereas in Institute 213 out of 38(34\%) patients of grade IV needed a cochleostomy.

Table I Inter group comparison of distribution of grades (grade wise)

\begin{tabular}{lll}
\hline Grade & & \\
\hline I & Chi-Square & II .645a \\
& Df & I \\
& Asymp. Sig. & $0.00 \mathrm{I}$ \\
2 & Chi-Square & I8.000b \\
& df & $\mathrm{I}$ \\
& Asymp. Sig. & 0 \\
3 & Chi-Square & $.054 \mathrm{c}$ \\
& df & $\mathrm{I}$ \\
& Asymp. Sig. & 0.816 \\
4 & Chi-Square & $\mathrm{I} 7.894 \mathrm{~d}$ \\
& df & $\mathrm{I}$ \\
& Asymp. Sig. & 0
\end{tabular}

\section{Discussion}

The soft surgery technique for hearing preservation in Cochlear Implantation has been the topic of discussion for few years. The round window approach has been advocated as a method to reduce insertion trauma and preserve residual hearing. However accessing the round window membrane could be difficult due to the bony overhang of the round window niche. Additionally the variable size and orientation of the round window may make electrode insertion difficult and challenging.

Round window electrode insertion has been noted for maximizing proper placement of the electrode in the scala tympani with evidences in the literature suggesting increased chances of scala vestibuli insertions and damage to the intra cochlear structures with more anteriorly placed conventional cochleostomy. ${ }^{5}$ The Round window membrane (RWM) is placed at around 900 angles to the oval window, has a bony over hang (niche) and is conical in nature with the horizontal postero-superior part which is closure to the osseous lamina and a vertical membranous antero-inferior part along which the electrode array is inserted..$^{11,12}$

The various factors which result in loss of residual hearing include surgical trauma during cochleostomy or during the insertion of the electrode (perilymph outflow, direct inner ear damage such as damage to the basilar membrane, osseous spiral lamina or lateral wall). There can be a delayed loss of residual hearing which is basically reactive in nature and is caused due to inner ear toxicity (blood, irrigation fluids or bone dust), or inflammation (infection, fibrosis, effusion) after placement of the electrodes. ${ }^{13}$ However, loss of residual hearing is primarily related to the event of insertion of the electrode array. ${ }^{14,15}$ Hence Lenhardt in 1993 proposed the soft surgery techniques for cochlear implantation in order to reduce this insertion related trauma. ${ }^{16}$

Round window membrane insertion is considered to be an important step in preservation of residual hearing as it minimizes intra cochlear trauma and subsequent new tissue formation. ${ }^{17,18}$ This is also less harmful compared to the standard cochleostomy. ${ }^{19}$ The better audiological outcomes with round window insertion are due to absence of drilling into the cochlea leading to prevention of chances of bone dust migration into the cochlea. ${ }^{4}$ Additionally, there is availability of increased length of spiral lamina averaging to $2 \mathrm{~mm}$ for stimulation with the electrode and utilisation of complete basal turn and increased perimodiolar placement of the electrode leading to increased stimulation of residual dendrites. ${ }^{5,20}$ Roland et al., ${ }^{21}$ demonstrated that drilling the overhand of the round window may increase the visualization by up to three times. This might allow the surgeon to insert the electrode along the midscala axis avoiding modular trauma.

In this study, an attempt has been made to classify the round window visibility before performing any drilling of the round window niche. This classification prepares the surgeon to have a prior idea about the extent of drilling that may be required after performing an adequate posterior tympanotomy. Our classification differs from the earlier classification by Leong et al., in which the authors graded the round window membrane after drilling the niche. The authors could not perform membranous insertion in $9 \%$ of cases of type IIb (less than $50 \%$ visibility) and all cases of type III (when no RWM could be seen even after best surgical drilling). The authors had to resort to the standard bony cochleostomy in such instances. They reported that drilling of the round window niche did not help in providing an access to the round window membrane and carried an inherent risk of drilling related trauma. On the contrary, the results suggests that it may be possible to do a membranous insertion in type II and III as surgeon has some idea about the extent of drilling of niche to expose the round window membrane fully.

We observed that around $65 \%$ cases with grade IV round window membrane. Eventually had a round window insertion. However, presence of a grade IV type of round window could caution the surgeon for a possible standard cochleostomy. We suggest that the Surgeon should always make an attempt to perform a round window insertion failing which a standard cochleostomy could be accomplished. For rest of the grades I, II and III; minimal drilling of postero-superior lip of round window niche and removal of cristae fenestra enabled us for membranous insertion of the electrode array into the cochlea, making our classification a more surgically viable one.

The proposed classification was validated by studying the distribution in two different geographical regions of the country. The distribution was similar in both the centres with regard to grade III type of round window ( $<25 \%$ or only glimpse of the round window being visible) being the commonest type of round window in Indian population. This validation in two centres was necessitated due to diversity of anthropometric variables in the two regions of the country. 22,23

\section{Conclusion}

Round window insertion is a significant step towards preservation of residual hearing during cochlear implant surgery. A reduction in the amount of drilling required for electrode placement during round window insertion is an important step for cochlear implant surgeons to achieve preservation of residual hearing. The proposed classification facilitates the surgeon to decide the amount of drilling that may be required to achieve a membranous insertion. It makes the decision easy to decide whether to attempt a membranous insertion or to go for bony cochleostomy. Grade III type of round window is the most common type of round window in Indian subcontinent which has been validated in two centres located in different geographical regions.

\section{Sources of financial support}

This research did not receive any specific grant from funding agencies in the Public, commercial or not for profit section.

\section{Acknowledgments}

None. 


\section{Conflicts of interest}

Author declares there are no conflicts of interest.

\section{Funding}

None.

\section{References}

1. Martini A, Bovo R, Trevisi P, et al. Cochlear implant in children: rational, indications and cost/efficacy. Minerva pediatrica. 2013;65(3):325-339.

2. Jolly C, Garnham C, Mirzadeh H, et al. Electrode features for hearing preservation and drug delivery strategies. Adv Otorhinolaryngol. 2016;67:28-42.

3. Garcia-Ibanez L, Macias AR, Morera C. An evaluation of the preservation of residual hearing with the Nucleus Contour Advance electrode. Acta oto-laryngologica. 2009;129(6):651-664.

4. Pau HW, Just T, Bornitz M, et al. Noise exposure of the inner ear during drilling a cochleostomy for cochlear implantation. The Laryngoscope. 2007;117(3):535-540.

5. Briggs RJ, Tykocinski M, Stidham K, et al. Cochleostomy site: implications for electrode placement and hearing preservation. Acta otolaryngologica. 2005;125(8):870-876.

6. Zanetti D, Nassif N, Redaelli de Zinis LO. Factors affecting residual hearing preservation in cochlear implantation. Acta Otorhinolaryngol Ital. 2015;35(6):433-441.

7. Chang A, Eastwood H, Sly D, et al. Factors influencing the efficacy of round window dexamethasone protection of residual hearing postcochlear implant surgery. Hear res. 2009;255(1-2):67-72.

8. Barriat S, Poirrier A, Malgrange B, et al. Hearing preservation in cochlear implantation and drug treatment. Advances in oto-rhinolaryngology. 2010;67:6-13.

9. Leong AC, Jiang D, Agger A, et al. Evaluation of round window accessibility to cochlear implant insertion. Eur Arch Otorhinolaryngol. 2013;270(4):1237-1242.

10. IBM SPSS Statistics for Windows [computer program]. Version 20.0. Armonk: IBM Corp, NY, USA. 2011.
11. Nomura Y. Otological significance of the round window. Advances in oto-rhino-laryngology. 1984;33:1-162.

12. Takahashi H, Sando I. Computer-aided 3-D temporal bone anatomy for cochlear implant surgery. The Laryngoscope. 1990;100(4):417-421.

13. Roland PS, Gstöttner W, Adunka O. Method for hearing preservation in cochlear implant surgery. Operative Techniques in OtolaryngologyHead and Neck Surgery. 2005;16(2):93-100.

14. Miranda PC, Sampaio AL, Lopes RA, et al. Hearing preservation in cochlear implant surgery. International journal of otolaryngology. 2014;2014(2014):468515.p.6.

15. Santa Maria PL, Gluth MB, Yuan Y, et al. Hearing preservation surgery for cochlear implantation: a meta-analysis. Otol Neurotol. 2014;35(10):e256-e269.

16. Lehnhardt E. Intracochlear placement of cochlear implant electrodes in soft surgery technique. HNO. 1993;41(7):356-359.

17. Havenith S, Lammers MJ, Tange RA, et al. Hearing preservation surgery: cochleostomy or round window approach? A systematic review. Otol Neurotol. 2013;34(4):667-674.

18. Richard C, Fayad JN, Doherty J, et al. Round window versus cochleostomy technique in cochlear implantation: histologic findings. 2012;33(7):1181-1187.

19. Nordfalk KF, Rasmussen K, Bunne M, et al. Deep round window insertion versus standard approach in cochlear implant surgery. Eur Arch Otorhinolaryngol. 2016;273(1):43-50.

20. Paprocki A, Biskup B, Kozlowska K, et al. The topographical anatomy of the round window and related structures for the purpose of cochlear implant surgery. Folia morphologica. 2014;63(3):309-312.

21. Roland PS, Wright CG, Isaacson B. Cochlear implant electrode insertion, the round window revisited. Laryngoscope. 2007;117(8):1397-1402

22. Majumder PP. People of India: Biological diversity and affinities. The Indian human heritage. 1999;8:45-59.

23. Bharati S, Demarchi D, Mukherji D, et al. Spatial patterns of anthropometric variation in India with reference to geographic, climatic, ethnic and linguistic backgrounds. Annals of human biology. 2005;32(4):407-444. 